\title{
An in vivo transposase-catalyzed single-stranded DNA circularization reaction
}

\author{
Patrice Polard and Michael Chandler \\ Laboratoire de Microbiologie et Génétique Moléculaires, UPR9007 du Centre National de la Resherche Scientifique (CNRS), \\ 31062 Toulouse CEDEX, France
}

\begin{abstract}
Expression of the bacterial insertion sequence IS911 transposase in vivo leads to excision and circularization of IS911-based transposons. We show here that transposase produces an unusual molecular form generated by single-strand cleavage, transfer, and ligation of one end of the element to the opposite end. When the transposon is carried by a circular plasmid, this results in the formation of a "figure-eight" molecule in which a single strand of the transposon is circularized while the corresponding strand of the vector backbone retains a single-strand interruption at this position. The results show that a $3^{\prime}$ end of the transposon is transferred to the opposite target end. Transposase is therefore capable of introducing single-strand cleavages at the ends of the element, an activity similar to that of retroviral integrases with which it shares significant similarities in amino acid sequence. Kinetic studies demonstrate that the figure-eight accumulates earlier than transposon circles after transposase induction and disappears before circles after inhibition of transposase expression, raising the possibility that the figure-eight molecules are precursors to the circles. Therefore, IS911 excision as a circle may not occur by double-strand cleavage leading to its prior separation from the vector backbone in a linear form but could proceed by consecutive circularization of each strand.
\end{abstract}

[Key Words: Transposase; circularization reaction; single-stranded; DNA; figure-eight molecule]

Received July 3, 1995; revised version accepted September 21, 1995.

During the past few years an increasing number of both eukaryotic and prokaryotic transposable elements have been observed to undergo excision accompanied by circularization (Morisato and Kleckner 1984; Rose and Snutch 1984; Ruan and Emmons 1984; Sundaresan and Freeling 1987; Scott et al. 1988; Jahn et al. 1989; Tausta et al. 1989; Polard et al. 1992; Radice and Emmons 1993; van Luenen et al. 1993; Williams et al. 1993; Sekine and Ohtsubo 1994). For many of these elements the circularization mechanism and its role in transposition remain to be elucidated. Such extrachromosomal circular forms have generally been thought to reflect a "cut and paste" transposition mechanism in which double-strand breakages liberate the element from the donor backbone and reciprocal strand exchange of transposase-bound ends then generates the circle (Caparon and Scott 1989; Polard et al 1992; Jaraczewski and Jahn 1993; Klobutcher et al. 1993; Williams et al. 1993; Sekine and Ohtsubo 1994).

Many elements that undergo circularization encode enzymes that are members of a recently defined super family of transposases (Doak et al. 1994). These enzymes exhibit a common motif composed of a triad of the acidic amino acids aspartate (D) and glutamate (E), the DD35E motif, first noted in retroviral integrases (INs) and certain bacterial transposases. Its function has been investigated in detail in retroviral integration, and it appears to form part of the active site of such enzymes. Current evidence indicates that integration of the dou- ble-stranded linear retroviral DNA copy is preceded by IN-mediated sequence-specific 3 ' single-strand breaks at the free retroviral ends (for reviews and an extensive bibliography, see Mizuuchi 1992; Skalka 1993; Vink and Plasterk 1993; Polard and Chandler 1995). Related enzymes encoded by DNA transposable elements may also introduce this type of $3^{\prime}$ single-strand break. In these cases, however, the elements are embedded in a donor DNA molecule. This raises the question of how the second 5' strand breaks necessarily involved in circle formation are generated.

One of the groups of circularizable elements whose transposases contain the DD35E motif is the IS3 family of bacterial insertion sequences (see Polard and Chandler 1995). Furthermore, like the proviral genome, the ends of these elements generally terminate with the $5^{\prime}-\mathrm{CA}-3^{\prime}$ dinucleotide. Members of the family show a similar genetic organization with two consecutive and partially overlapping open reading frames, orf $A$ and orf $B$, flanked by short imperfect terminal repeats, IRL (left end) and IRR (right end) (Schwartz et al. 1988; Prère et al. 1989|. Three of these elements, IS3, IS150, and IS911, have each been shown to encode three proteins from the two open reading frames. The transposase is a fusion protein, OrfAB, produced by a programmed -1 translational frameshifting between the two frames, leading to the combination of the DD35E motif, encoded by the orf $B$ frame (Fayet et al. 1990) and of a potential $\alpha$-helix- 
turn- $\alpha$-helix DNA-binding domain, located in $\operatorname{orf} A$, into a single protein (Prère et al. 1989). When provided with transposase alone in vivo, these three insertion sequences have been shown to form double-stranded circular copies in which IRL and IRR are abutted (IS911, Polard et al. 1992; IS3, Sekine and Ohtsubo 1994; IS150, C. Weltz-Vögele and B. Rak, pers. comm.).

Studies on the mechanism of IS911 circularization have led to the proposal that it results from the transfer of a single end of the element to the other in a sitespecific manner (Polard et al. 1992). One copy of the 3-bp target duplication generated on IS 911 insertion is retained between the abutting IRL and IRR ends on the circle, although neither the nucleotide sequence of this flanking duplication nor the duplication itself appears important for circularization. Excision of an IS911 derivative in which the sequences flanking IRL and IRR are not identical results in a mixed population of circles, carrying either one of the flanking $3 \mathrm{bp}$ between the abutted ends. Furthermore, although mutation of the conserved terminal 5'-CA-3' dinucleotide at both ends abolishes circularization, an element carrying only one mutated end can still circularize giving a population of circles in which the intervening $3 \mathrm{bp}$ are exclusively those bordering the mutated end. This demonstrates the importance of the terminal 5'-CA-3' dinucleotide and also suggests that circularization results from a polarized transfer of the wild-type end to the mutant end. Precise circularization of IS911 appears to be a one-ended intramolecular transposition event in which one end acts as a site-specific target (Polard et al. 1992). Although these data have provided a general picture of how IS911 circles are generated, they do not permit a precise description of the excision mechanism. In particular, it remains unclear how strand cleavages occur at the transposon ends and whether these cleavages are consecutive or concerted.

In the results presented here we describe a second major molecular species whose appearance, like that of the transposon circles, is also transposase dependent. It results from the transfer of a single strand of one transposon end 3 nucleotides from the other end without breakage of the second strand. Therefore, the initially circular plasmid donor molecule is converted to a nicked "figureeight" form in which a single transposon strand is circularized with the three extraneous nucleotides bridging the two ends. The use of elements mutated for their terminal dinucleotides has demonstrated that like circularization, this bridged form is the result of a one-ended intramolecular transposition reaction. The results strongly support the idea that transposition occurs by transfer of a $3^{\prime} \mathrm{OH}$ transposon end into the target site, as has been demonstrated for several other elements. The structural similarity between the figure-eight and circle suggests that the bridged product could be an intermediate in circularization. Both kinetic and stability analyses of the figure-eight compared to that of the circle are consistent with this idea. Therefore, IS911 circularization may not involve a step in which the element is excised in a linear form.

\section{Results \\ Experimental system}

To study intermediate steps in IS 911 transposition in vivo, we have assembled a two-plasmid system that permits induction and amplification of the process in a controlled manner. One plasmid serves as a source of transposase, Orf $A B$, and carries the transposase gene, whereas the other carries a transposon substrate (Fig. 1). Transposase expression is driven by the IPTG-inducible lac promoter that is controlled by a lac $I^{\mathrm{q}}$ gene present on the same plasmid. The transposase gene is present as a 1206bp fragment of IS911 in which the terminal 22 bp of each end have been removed but in which the weak indigenous translation initiation signals of orf $A$ have been retained. Plasmids carrying a mutant in which orf $A$ and orf $B$ are fused artificially, pAPT111, or wild-type coding sequences, pAPT112, were used as sources of transposase. The mutations introduced into the gene carried by pAPT111 ensure contiguous translation of OrfAB (i.e., without frameshifting) and eliminate expression of the OrfA and OrfB proteins (Polard et al. 1991). The plasmid
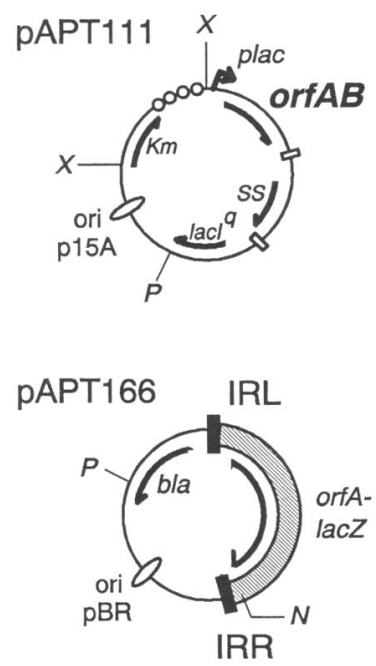

Figure 1. Structure of IS 911 transposase and transposon donor plasmids. The transposase donor plasmid, pAPT111 (8028 bp), is a p15A-based replicon. The compatible transposon donor plasmid pAPT166 (6851 bp) is a pBR322-based replicon. Relevant restriction sites are indicated: $(\mathrm{N}) \operatorname{NdeI}_{;}(\mathrm{P}) \operatorname{Pst}_{\mathrm{I}}(\mathrm{X})$ Xhol. Open ovals indicate the origin of replication. Open circles indicate $r r n B$ transcription terminators, and open boxes are phage $\mathrm{T} 4$ gene 32 transcription terminators. Relevant genes are indicated together with their direction of transcription within the plasmid circle: $(\mathrm{Km})$ resistance to kanamycin; (SS) resistance to streptomycin and spectinomycin; (bla) $\beta$-lactamase. The $1 a I^{q}$ allele is present in pAPT111 to control the lac promoter, plac, which in turn drives IS911 orf $A B$ gene expression. The mutation that fuses the orf $A$ and $\operatorname{orf} B$ frames to generate orf $A B$, is from AUUA $_{6} \mathrm{G}$ (pAPT112) to $\mathrm{ACUCA}_{6} \mathrm{G}$ (pAPT111). The IS911based transposon carried by pAPT 166 is shown in boldface type. Solid boxes represent the left (IRL, $86 \mathrm{bp}$ ) and right (IRR, $51 \mathrm{bp}$ ) terminal inverted repeats. The orf $A-\beta$-galactosidase gene fusion is indicated within these repeats. 
that contributes the transposable element, pAPT166, is a derivative of pAT153, which is compatible with the transposase donor plasmid. It carries an artificial $3.3-\mathrm{kb}$ IS911-based transposon composed of $86 \mathrm{bp}$ of the left end (IRL), which includes the first 8 codons of orf $\mathrm{A}$, and a flanking 51-bp right end (IRR). The orfA sequences are fused translationally with a $l a c Z$ gene.

As shown in Figure 2, DNA prepared by alkaline lysis of overnight IPTG-induced cultures of cells carrying both pAPT111 and pAPT166 (lane 4) exhibits an additional species smaller than the parental plasmids. Hybridization, restriction analysis, electron microscopy (data not shown), PCR, and nucleotide sequence determination (see below) demonstrate that this corresponds to a supercoiled circular form of the transposable element similar to that observed previously (Polard et al. 1992). Although detectable in the absence of IPTG (lane 2 ), it is present at higher levels in cultures in which transposase expression is induced (lane 4). Therefore, these results demonstrate that the system functions in the inducible production of transposon circles.

\section{A novel transposase-induced molecular species}

The procedure used to prepare plasmid DNA in the pre-

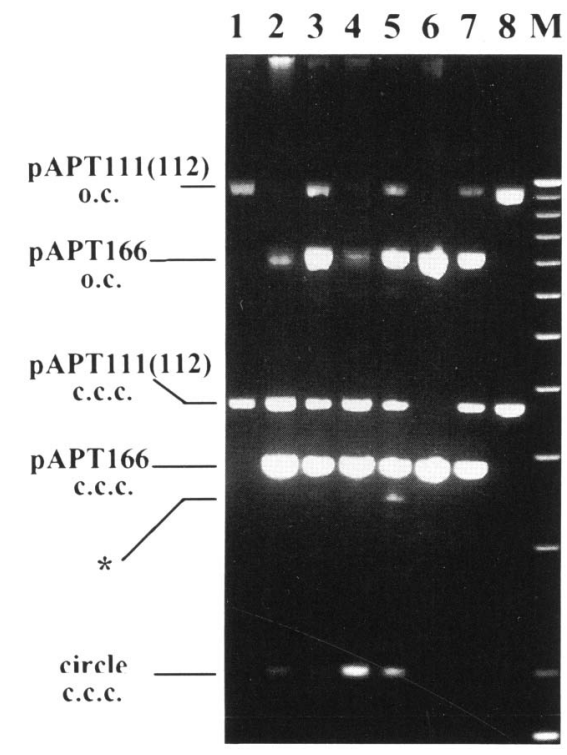

Figure 2. A novel IS 911 transposase-induced molecular species. DNA samples were prepared either by alkaline lysis (lanes 2,4 ) or a cleared lysate procedure (lanes 1, 3, 5-8) and separated on a $0.7 \%$ agarose gel (in TAE buffer). (Lanes $1,6,8)$ pAPT111 (fused frame-transposase donor), pAPT166 (transposon donor), and pAPT112 (wild-type orfA and orfB) alone with IPTG; (lanes 2, 3) pAPT11l and pAPT166, no transposase induction, alkaline and cleared lysate, respectively; (lanes 4, 5) pAPT111 and pAPT166, with transposase induction, alkaline and cleared lysate, respectively; (lane 7) pAPT112 and pAPT166 with transposase induction, cleared lysate; (lane M) 1-kb ladder markers. Position of open circular (o.c.) and closed circular (c.c.c.) forms of the parental plasmids are indicated at left as are those of the closed circular form of the transposon circle and the novel form $(*)$. vious experiments involved a denaturing step. It seemed possible that additional DNA species produced by the action of OrfAB, but that are not closed circular, might have been overlooked. Therefore, DNA was prepared using a less aggressive "cleared lysate" method (Clewell and Helinski 1969) from cells carrying both pATP111 and PAPT166 after overnight growth in the presence of IPTG (Fig. 2). In addition to the closed circular transposon form detected after alkaline lysis (cf. lanes 2 and 4 with lanes 3 and 5 ), an additional band migrating slightly ahead of the transposon donor plasmid, pAPT166, can be observed (lane $5, *$ ). As in the case of circle formation, this species does not appear when transposase is supplied from wild-type coding sequences (pAPT112, lane 7). Southern hybridization indicated that the novel band exhibits homology to both the transposon and vector backbone segments of pAPT166 (data not shown). Therefore, it cannot represent either an open circular or a linear form of the transposon separated from the donor plasmid backbone. Furthermore, extensive protease digestion (see Materials and methods) included in the extraction procedure makes it unlikely that the new species is a protein-bound DNA structure. One additional characteristic of this band is that its migration, relative to the other DNA species, is very sensitive to the field strength during electrophoresis (data not shown). This suggests that it could represent a complex topological form of the transposon donor plasmid generated by the transposase.

\section{Molecular characterization of the new species: a nicked figure-eight molecule}

The data presented below demonstrate that the new DNA species is a figure-eight molecule in which a single strand of the transposon is circularized [Fig. 3a(i)].

\section{Restriction analysis and electron microscopy}

Total plasmid DNA samples were digested with the restriction enzymes PstI and NdeI, which have single cleavage sites in the plasmid backbone and in the transposon of pAPT166, respectively (see Fig. 1), and with XhoI, which cleaves twice within pAPT111 but not within pAPT166.

Digestion of plasmid DNA isolated from the strain carrying pAPT111 and pAPT166 with XhoI and NdeI [Fig. 3b(i)] reveals two additional bands (lane 2) compared with those generated with pAPT111 (lane 1) and pAPT166 (lane 3) alone. The lower of these two bands migrates as expected for the transposon circle linearized by cleavage at the single NdeI site $(3.3 \mathrm{~kb})$. The upper band derives from the novel DNA species, as it exhibits an identical apparent molecular weight to that obtained from digestion of a purified preparation of this species with NdeI alone (lane 4). The results of digestion of these samples with $X$ hoI and $P s t \mathrm{I}$ is presented in Figure $3 \mathrm{~b}(\mathrm{ii})$. Again, DNA isolated from the strain carrying both pAPT111 and pAPT166 shows two additional bands (lane 2) compared with those generated with pAPT111 (lane 1) and pAPT166 (lane 3) separately. The lower of 
a)

(i)

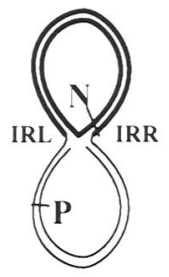

(ii)
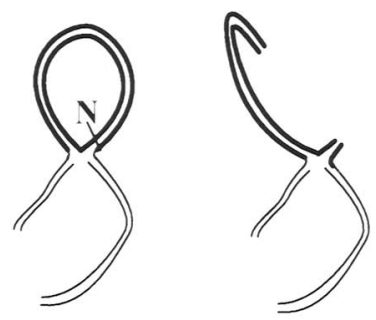

b)

(i)

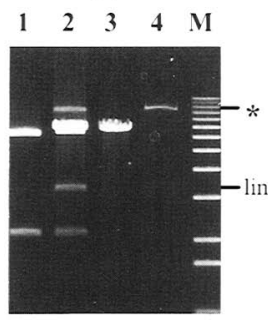

(ii)

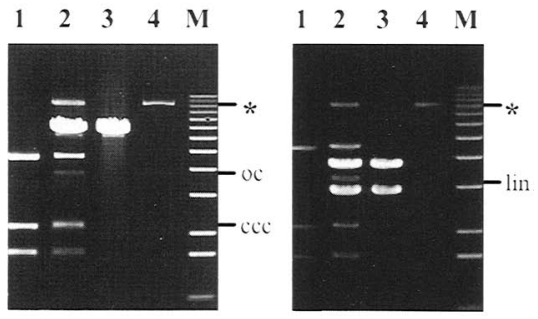

Figure 3. Restriction analysis of transposase-induced species. (a) Diagrams showing proposed structures of complete and digested species. Heavy lines represent transposon DNA, lighter lines represent vector backbone. NdeI $(\mathrm{N})$ and $P_{s} t \mathrm{I}(\mathrm{P})$ sites are indicated with respect to the terminal inverted right and left repeats (IRR and IRL). (i) Undigested; (ii) PstI digested; (iii) PstINdeI digested. (b) Digestion and fractionation of DNA samples. Fragments were separated by electrophoresis on a $1 \%$ TAE agarose gel. (Lane 1) pAPTlll alone; (lane 2) pAPT11l and pAPT166; (lane 3) pAPT166 alone; (lane 4) purified novel DNA species; (lane $M$ ): 1-kb ladder markers. The position of open and closed circular forms of the transposon circle, together with the new form $\left({ }^{\star}\right)$, are shown at right. (i) PstI digested; (ii) NdeI digested; (iii) PstI-NdeI digested.

the two bands is an open circular (o.c.) form of the transposon circle. It should be noted that the supercoiled form of the circle (c.c.c.) comigrates with a XhoI fragment of the transposase donor plasmid pAPT111. The upper band is derived from the new DNA species and migrates identically to that obtained from digestion of a purified preparation of this species with PstI alone (lane 4). One important feature of the upper band is that in both cases it migrates more slowly than linearized donor plasmid DNA [Fig. 3b(i and ii), cf. lanes 3 and 4]. Like the new species in its undigested form, the migration of these bands relative to the others also varies with the field strength applied during electrophoresis (data not shown). When digested with all three enzymes, the new species continues to migrate as a single high molecular weight band [Fig. 3b/iii), lanes 2,4] similar to those obtained with NdeI-XhoI or PstI-XhoI.

These restriction patterns and electrophoretic mobilities are consistent with a structure in which the transposon donor plasmid is bridged by a covalent DNA linkage between the two transposon ends generating a figure- eight configuration [Fig. 3a(i)]. A single cleavage in the transposon domain (NdeI) or in the vector plasmid backbone (PstI) would each yield a molecule with an $\alpha$ form [Fig. 3a(ii)]. Such species would be expected to exhibit different migration properties from linearized plasmid DNA. Similarly, digestion with both NdeI and PstI would give rise to molecules with a $\chi$ form rather than two DNA fragments [Fig. 3a(iii)].

Further support for these structures was obtained by electron microscopy of the respective bands purified from the agarose gel. Figure-eight forms were observed among the native molecules (Fig. 4a). Although, for clarity the structure shown here is relaxed, it should be noted that the majority of molecules in this preparation were supercoiled. Molecules isolated after digestion with Pst I alone exhibited a majority species in the form of an $\alpha$ (Fig. 4b) in which the arms were estimated to be $795 \pm 78$ and $2799 \pm 72$ bp long and the circular section, $3288 \pm 68$ bp $(n=25$ in all cases). The predicted lengths for a structure in which the branchpoint occurs at the transposon ends are 810 and $2761 \mathrm{bp}$, and the circular transposon section, $3283 \mathrm{bp}$, in good agreement with the observed values.

DNA isolated after digestion with both PstI and NdeI showed a majority of molecules in the form of a $\chi$ (Fig. 4c). Again, two arms of $777 \pm 54$ and $2726 \pm 72$ bp have a length expected for those generated by PstI $(810$ and 2761 bp), whereas those presumably generated by NdeI have lengths of $172 \pm 12$ and $2998 \pm 53 \mathrm{bp}(n=25)$, in good agreement with that expected (223 and $3057 \mathrm{bp}$ ).

The electron micrographs suggest that the bridge of the figure-eight is single-stranded rather than doublestranded. This conclusion is reinforced by the observation that two linear DNA fragments were sometimes observed in preparations of the $x$ form. These have lengths of $2781 \pm 82$ and $3729 \pm 67 \mathrm{bp}(n=25)$ and could arise from breakage of a single-strand linkage between IRR and IRL (expected sizes 2984 and 3867 bp).
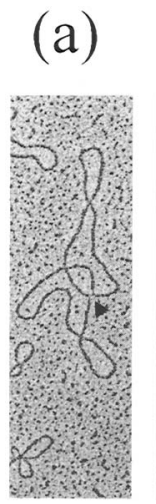

(b)

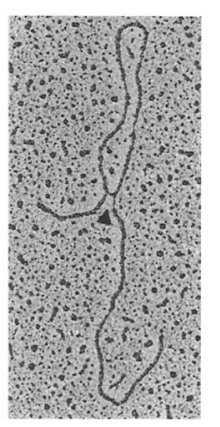

(c)

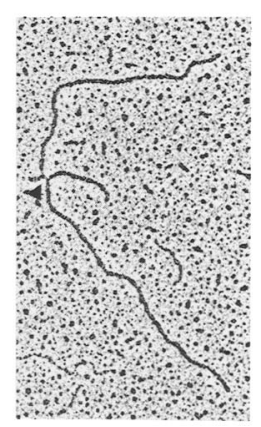

Figure 4. Electron microscopy of purified native and digested novel species. Representative molecules of figure-eight species derived from pAPT166 and purified after agarose gel electrophoresis are shown. The arrowheads point to the single-stranded bridge. (a) Undigested; $(b)$ PstI digested; (c) PstI-NdeI digested. Magnification is identical in all three cases. 


\section{PCR analysis}

Electron microscopy indicates that the population of recombined molecules is quite homogenous and locates the new junction close to the IS 911 terminal inverted repeats, IRR and IRL. Such a junction could be generated by a single-strand transfer resulting in circularization of one or the other of the transposon strands and leaving a single-strand nick in the vector backbone (Fig. 5a).

To determine the nature and position of the junction more precisely, the structure of the figure-eight molecule was probed using a PCR approach. The analysis was performed on a gel-purified $\alpha$ form generated by PstI or NdeI digestion of the figure-eight. With PstI digestion the transposon domain should remain intact and the plasmid backbone will generate two arms, whereas with $N d e I$ the plasmid backbone should remain intact and the transposon should generate two arms. A combination of four oligonucleotides, $\mathrm{O}_{0}, \mathrm{O}_{3}, \mathrm{O}_{5}$, and $\mathrm{O}_{7}$ was used (Fig.

a)

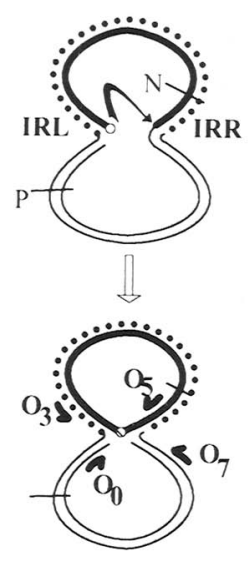

b)

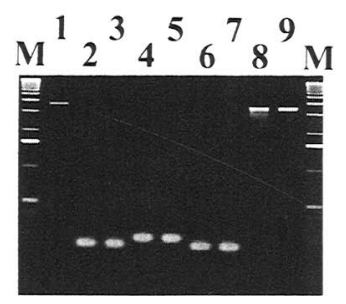

Figure 5. PCR analysis. (a). Single-strand transfer resulting in circularization of one transposon strand. Transposon DNA is shown as heavy lines. Continuous and dotted lines indicate the two DNA strands. The open circle represents the transferred (arrow) 3' transposon end. The two strands of vector DNA are drawn as continuous thin lines. (Bottom) The relative positions of oligonucleotides, $\mathrm{O}_{0}, \mathrm{O}_{3}, \mathrm{O}_{5}$, and $\mathrm{O}_{7}$. Symbols are the same as in Fig. 1. (b) The results of PCR analysis using $1.2 \%$ TEAagarose gel electrophoresis: $\mathrm{O}_{3}$ and $\mathrm{O}_{5}$ (lanes 1-3), $\mathrm{O}_{0}$ and $\mathrm{O}_{3}$ (lanes 4, 5), $\mathrm{O}_{5}$ and $\mathrm{O}_{7}$ (lanes 6, 7), $\mathrm{O}_{0}$ and $\mathrm{O}_{7}$ (lanes 8, 9). (Lane M) 1-kb ladder markers. The DNA matrices used are pAPT166 (lanes $1,4,6,8$ ); purified $\alpha$ form generated by NdeI digestion (lane 2, 5, 7); purified $\alpha$ generated by PstI digestion (lane 9); purified transposon circle (lane 3 ). 5a). Each hybridizes with one of the four arms suspected to be crossed on the figure-eight. $\mathrm{O}_{\mathrm{O}}$ is complementary to sequences bordering IRL and is directed toward IRL. $\mathrm{O}_{3}$ hybridizes with an IS 911 sequence close to IRL and is directed toward $\mathrm{O}_{0}$. Oligonucleotides $\mathrm{O}_{5}$ and $\mathrm{O}_{7}$ are equivalent to $\mathrm{O}_{3}$ and $\mathrm{O}_{0}$, respectively, on the IRR side of the molecule (Fig. 5a).

$\mathrm{O}_{3}$ and $\mathrm{O}_{5}$ generate a fragment of $3.7 \mathrm{~kb}$ with the transposon donor plasmid pAPT166 as a matrix (Fig. 5b, lane 1 ), and a characteristic fragment of $\sim 150 \mathrm{bp}$ carrying abutted IRR and IRL ends is obtained with the transposon circle (lane 3). The figure-eight molecule ( $\alpha$ cleaved by NdeI in the transposon domain) gives rise to a fragment that comigrates with that obtained using the transposon circle as a matrix rather than the large fragment generated by the donor plasmid (lane 2). The figure-eight form thus carries abutted copies of IRL and IRR. Oligonucleotides $\mathrm{O}_{0}$ and $\mathrm{O}_{3}$ generate a fragment of $200 \mathrm{bp}$ with both pAPT166 (lane 4) and the NdeI-cleaved figureeight form (lane 5), whereas $\mathrm{O}_{5}$ and $\mathrm{O}_{7}$ generate a fragment of $150 \mathrm{bp}$ with both molecules (lanes 6, 7). This indicates that in addition to the new IRL/IRR junction, both parental IRL and IRR plasmid junctions are retained in the figure-eight form. Oligonucleotides $\mathrm{O}_{0}$ and $\mathrm{O}_{7}$ generate a fragment of $3.3 \mathrm{~kb}$ with both donor plasmid and the figure-eight form $/ \alpha$ cleaved by PstI in the vector domain; lanes 8,91 . This corresponds to amplification of the entire transposon and demonstrates that the plasmid backbone sequences are not involved in formation of the new junction. Finally, the absence of any PCR fragment from the NdeI-cleaved figure-eight with the pairs $\mathrm{O}_{0}-\mathrm{O}_{5}$ and $\mathrm{O}_{3}-\mathrm{O}_{7}$ (data not shown) indicates that IRL is not connected to the IRR flanking sequences, nor IRR to the sequences flanking IRL.

Together these experiments demonstrate that the donor plasmid pAPT166 is converted into a specific figureeight form by circularization of a single transposon strand. This leaves the adjacent plasmid DNA strand unjoined and the complementary transposon strand connected to the plasmid backbone as shown in Figure 5a.

\section{The mechanism of figure-eight formation}

The results presented above do not address the question of how strand cleavage occurs, which transposon strand is transferred, or the mechanism of strand transfer itself. Answers to these questions require the separation of the individual reaction steps.

\section{The figure-eight results from a single $3^{\prime}$ strand transfer event}

Analysis of IS911 circle formation has indicated that the terminal $5^{\prime}$-CA-3' dinucleotide is important for strand cleavage or transfer (Polard et al. 1992). Mutation of the terminal $5^{\prime}-\mathrm{CA}-3^{\prime}$ was found to exhibit similar effects on the formation of figure-eight molecules. Analysis of plasmid DNA samples prepared after overnight growth of induced cultures of cells carrying the donor plasmid, pAPT182, with mutations in both ends of the transposon 
did not generate figure-eight molecules, whereas plasmids carrying mutations in either IRL (pAPT180) or IRR (pAPT181) retained the capacity for figure-eight formation (data not shown). The nucleotide sequence of the figure-eight junctions amplified by PCR using oligonucleotides $\mathrm{O}_{3}$ and $\mathrm{O}_{5}$ (see Fig. 5a) was identical to that of the equivalent circular products; they all carry abutted IRL and IRR ends separated by $3 \mathrm{bp}$. The 3 -bp spacer derived from the wild-type donor (pAPT166) was composed of sequences flanking both wild-type ends, whereas for those derived using a single mutant end, the spacer was composed exclusively of sequences adjacent to that end (data not shown). Thus, as in the case of circle formation (Polard et al. 1992), a simple hypothesis to explain the mixed spacer sequence of the junction fragment PCR-amplified from wild-type figure-eight molecules is that the population is composed of two symmetrical molecules determined by which transposon strand undergoes circularization.

Only a single cleavage and strand transfer event are required to generate figure-eight molecules. Therefore, the nucleotide sequence of the single-strand junction of figure-eight molecules should define the polarity of the strand cleavage and transfer reactions. This was determined by direct sequencing of figure-eight molecules from single end mutants using oligonucleotide $\mathrm{O}_{2}$ (Fig. 6). This is complementary to IRL and is directed out of the transposon toward the vector backbone. The sequence generated with the mutant IRR (Fig. 6, top) indicates that the $5^{\prime}$ end of IRL is contiguous with the flanking donor backbone, whereas that generated with the mutant IRL is contiguous with IRR (Fig. 6, middle). The nucleotide sequences obtained, complementary to the the continuous heavy strands, are presented below the diagrams. Inactivation of one transposon end by mutation thus reveals a polarized single-end transfer. As expected the figure-eight population derived from a transposon with two wild-type ends is composed of both figure-eight orientations. This is characterized by an IRL sequence contiguous with the original bordering $3^{\prime}$ parental nucleotides and is followed by a double (IRR and plasmid backbone) nucleotide sequence (Fig. 6, bottom). Opposite and symmetric results were obtained using the IRR-specific oligonucleotide, $\mathrm{O}_{5}$ (data not shown). Therefore, these results strongly support the idea that a single transposon strand is involved in the reaction and, moreover, that it is a $3^{\prime}$ end that is transferred 3 nucleotides $5^{\prime}$ to the opposite end.

\section{Mapping of the vector backbone strand interruption}

Electron microscopy and PCR analysis suggest that the vector backbone is not joined at the IRL/IRR junction in the figure-eight molecules. An important aspect of the predicted figure-eight structure is that the strand interruption should be located at the site where the $3^{\prime}$ strand transfer reaction has occurred. To demonstrate and map this presumed single-strand nick, primer extension with oligonucleotide $\mathrm{O}_{0}$ was performed on the three types of figure-eight molecules (i.e., both single-end mutants and
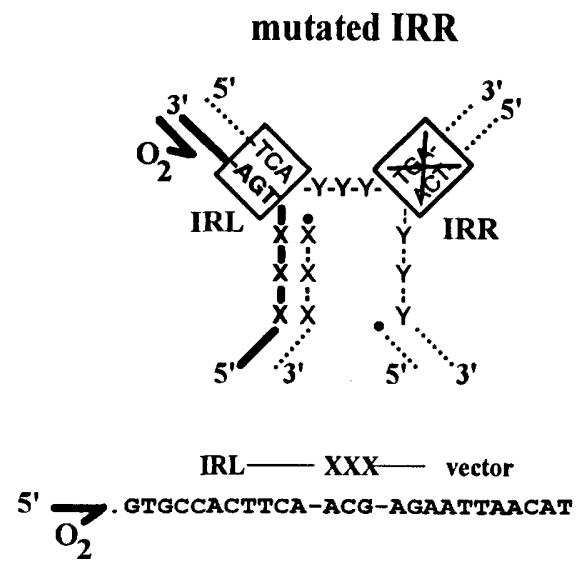

mutated IRL
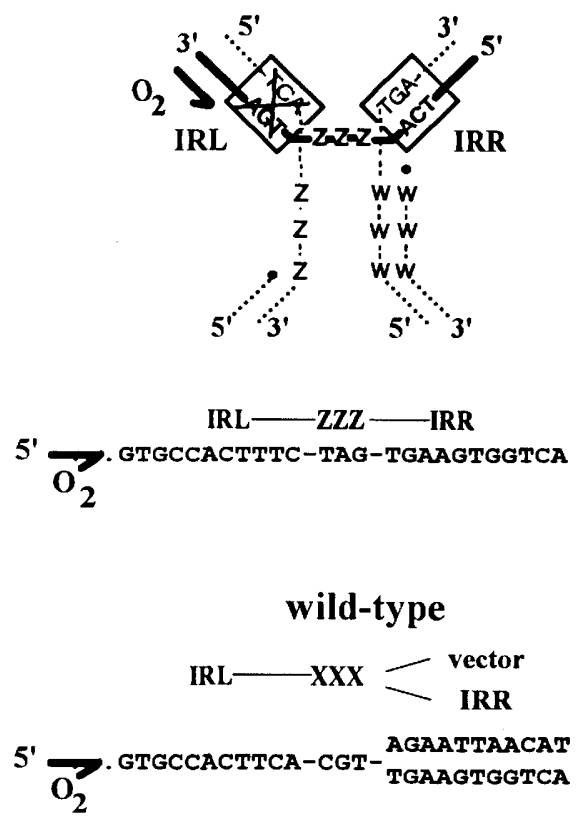

Figure 6. Nucleotide sequence at the crossover point of the figure-eight. (Top) The deduced structure of the single-stranded bridge obtained with a transposon in which the right end (IRR) has been inactivated by mutation. (Middle) An equivalent structure for mutation of the left end. The position of oligonucleotide $\mathrm{O}_{2}$, used as a primer for determining the nucleotide sequence at the junction, is indicated. The continuous template strand is shown in boldface type. The ends of the transposon are boxed, and the mutated end is indicated by a cross within the box. The transposon was designed to carry different distinguishable flanking 3 bp. For clarity, these have been designated XXX, YYY, ZZZ, and WWW. The sequence of the mutated left and right ends was $5^{\prime}-\mathrm{TC}-3^{\prime}$ and $5^{\prime}$-AG-3', respectively. The nucleotide sequence of the IRL junction of gel-purified figure-eight molecules is shown under each diagram. Plasmids are pAPT1 180 (mutated IRL) and pAPT181 (mutated IRR). These sequences represent the complementary sequence of the continuous bold strand. (Bottom) The mixed sequence obtained with pAPT166 (wild-type ends). 
the wild-type substrate). The rationale of this experiment is shown in Figure 7. Oligonucleotide $\mathrm{O}_{0}$ is complementary to the vector backbone flanking IRL and can be extended toward the junction. Purified figure-eight substrates were first digested with Ball, which cleaves within IRL. This provides an artificial strand interruption as an internal control for primer extension because the site is located downstream from the expected point of vector strand breakage.

Primer extension of figure-eights isolated from the IRR mutant results in a single band ending precisely before the first nucleotide of IRL (Fig. 7, top). A majority of molecules carries this specific nick as no band corresponding to the upstream Ball site can be detected.

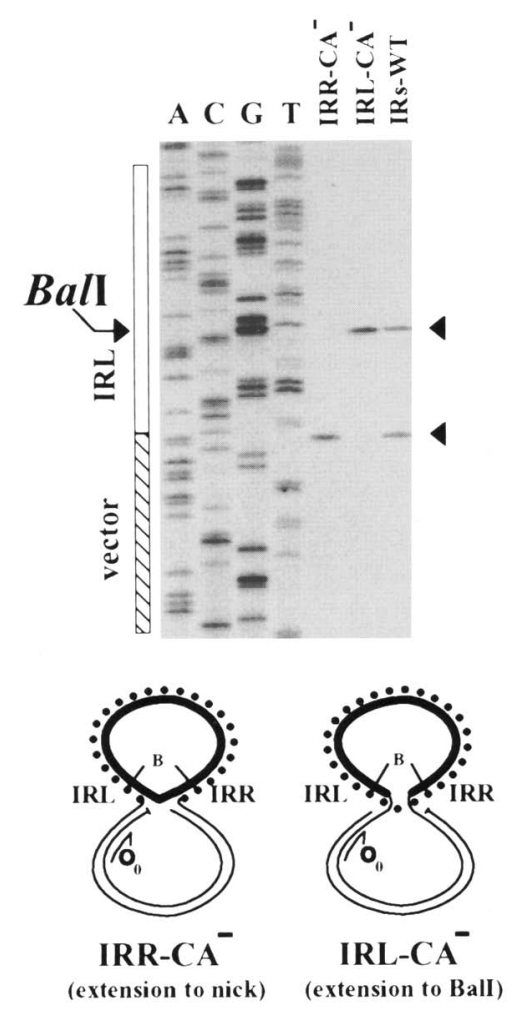

Figure 7. Localization of the strand interruption in the vector backbone. (Top) The results of primer extension using oligonucleotide $\mathrm{O}_{0}$, from the vector backbone (crosshatched segment at left) toward the IRL end of transposon DNA (open segment). The position of the Ball site is indicated. The nucleotide sequence shown corresponds is that obtained from the parental plasmid using the same oligonucleotide. All substrates were cleaved with BalI before to primer extension. (IRR-CA ${ }^{-}$) Figure-eights isolated from pAPT181 (mutant IRR); (IRL-CA ${ }^{-}$) pAPT180 (mutant IRL); (IRs-WT) pAPT166 (wild-type ends1.(Bottom) The experimental design. The symbols are identical to those in the drawing in Fig. 4a. Note that both IRL and IRR carry a BalI (B) site. The approximate position of the oligonucleotide $\mathrm{O}_{0}$ is shown. (Left) The expected conformation of a figure-eight derived with a mutated IRR: Extension would terminate at the cleaved vector and not reach the BalI site. (Right) The expected conformation of a figure-eight derived with a mutated IRL: Extension would terminate at the BaII site.
Primer extension of figure-eights isolated from the IRL mutant yields a band corresponding exclusively to termination at the Ball site indicating the absence of a nick at the mutated IRL/vector junction. The specific nick in this case must be located at the (wild-type) IRR/vector junction. An identical experiment with the wild-type figure-eight molecules generates both signals with approximately equal intensity, implying that only half the population of molecules carries a specific nick at the (wildtype) IRL junction. Therefore, the results provide further evidence that the wild-type transposon is converted to a mixture of figure-eight molecules of both configurations. They also demonstrate that one strand of the vector backbone is nicked and that the nick is located exactly at the $3^{\prime}$ end of the transposon.

\section{Nature of the nick in the vector backbone}

If single-strand circularization involves generation of a $3^{\prime} \mathrm{OH}$ at one transposon end and subsequent attack by that end of a $5^{\prime}$ phosphate group of the target, the strand transfer reaction should leave a $3^{\prime} \mathrm{OH}$ group on the appropriate vector strand bordering the target (Fig. 8, bottom/, which should be capable of acting as a primer in strand extension. To examine this, figure-eight molecules generated from the plasmids carrying the terminal base pair mutations in IRL (PAPT180) or in IRR (pAPT181) were isolated, digested with both BalI and EcoRI, subjected to strand extension, and analyzed on a sequencing gel. Extension of the recessed $3^{\prime} \mathrm{OH}$ end generated by EcoRI provides an internal marker in this experiment (Fig. 8, bottom). In the IRL mutant, exclusive strand transfer of the wild-type IRR end $5^{\prime}$ to the mutant end should generate a free $3^{\prime} \mathrm{OH}$ on the bordering vector DNA at this position (close to the EcoRI site; Fig. 8, bottom). The end should be located outside the transposon, $3 \mathrm{bp} 5^{\prime}$ to the terminal IRL base pair. Extension of this strand by 22 bases to the Ball generated end would produce a strongly labeled fragment of 82 bases. Note that the extended $3^{\prime}$ end of the cleaved EcoRI site $(4$ bases) will also provide a signal of an identical length for the complementary strand. Under conditions of continuous labeling used here in strand extension, this signal is expected to be weaker than that of the complementary strand because of the short extension at the EcoRI site. In the IRR mutant, only the IRL end is transferred. In this case, the strand interruption in the vector DNA proximal to the EcoRI site should result in a $5^{\prime}$ end that will not be extended (Fig. 8, bottom). This strand can, however, be extended from the $3^{\prime}$ EcoRI-generated end, resulting in a weakly labeled shortened 60-base fragment not observed with the IRL mutant. Figure-eight molecules from the wild-type transposon (pAPT166) should exhibit a mixture of these signals. The results of such an experiment are presented in Figure 8 (top). As predicted, the IRR mutant exhibits only the short 60 -base weakly labeled fragment, the IRL mutant produces a strong signal corresponding to the expected 82-base fragment. The wild-type figure-eight substrate gives rise to a mixture of both signals with the expected bias in labeling of the larger fragment. 


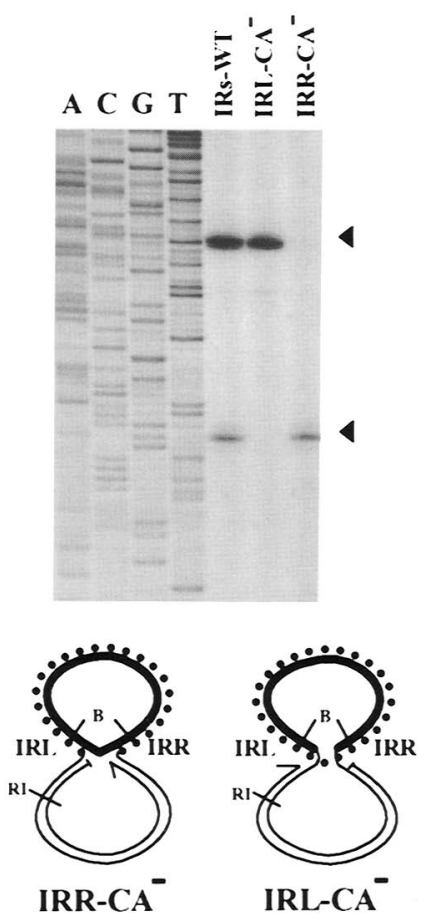

Figure 8. The strand interuption in the vector backbone carries a $3^{\prime} \mathrm{OH}$. (Top) The results of extension from the vector backbone toward the IRL end of transposon DNA. The nucleotide sequence shown is that of M13mp18 using the universal primer. It was included as a size marker. All substrates were cleaved with Ball and EcoRI before extension. (IRR-CA- / Figure-eights isolated from pAPT181 (mutant IRR); (IRL-CA ${ }^{-}$) pAPT180 (mutant IRL); (IRs-WT) pAPT166 (wild-type ends). (Bottom) The experimental design. The symbols are identical to those in the drawing shown in Fig. 7. The relative position of the EcoRI (RI) and Ball (B) sites are indicated. The vector $3^{\prime} \mathrm{OH}$ end is shown as a curved half-arrow. (Left) The expected conformation of a figure-eight derived with a mutated IRR. (Right) The expected conformation of a figure-eight derived with a mutated IRL.

Therefore, these results demonstrate that the target of the $3^{\prime}$ strand transfer of one transposon end is a $5^{\prime}$ phosphate at the other end. This leaves a $3^{\prime} \mathrm{OH}$ group on the neighboring cleaved vector DNA at the target site.

\section{Is the figure-eight the precursor to the circle?}

The structure of the figure-eight molecule clearly raises the possibility that it is an intermediate in the formation of transposon circles. To explore this possibility we have examined the kinetics of formation and the stability of both species.

\section{Kinetics of figure-eight and circle production}

A culture of cells carrying both the transposase donor, pAPT111, and the transposon donor, pAPT166, was induced for transposase synthesis in mid-log phase. DNA samples prepared at various times (see Materials and methods) were digested with XhoI, PstI, and NdeI and subjected to agarose gel electrophoresis. The gel was dried and probed with end-labeled oligonucleotide $\mathrm{O}_{2}$. This is complementary to the IS911 sequence adjacent to IRL present in pAPT111, pAPT166, the transposon circle, and figure-eight products and permits detection of all four types of molecule. To obtain a more precise measure of the kinetics of appearance of the linearized transposon circles and $\chi$ forms, the intensity of the respective bands (Fig. 9a, top) were normalized to total plasmid DNA in each sample and plotted as a function of time after transposase induction (Fig. 9a, bottom). Although there is a measurable level of both figure-eight and circle species before induction (Fig. 9a, top, lane 1), after transposase induction, the band representing the figure-eight species clearly increases in intensity before the transposon circles.

Accumulation of figure-eight molecules begins rapidly immediately after transposase induction and then slows after $\sim 45 \mathrm{~min}$, at a time when the level of the circular species begins to increase.

\section{Stability of the figure-eight and transposon circles}

The kinetics of appearance of figure-eight molecules with an initial rapid rate of accumulation followed by a gradual reduction might be explained by the establishment of an equilibrium between their rates of synthesis and breakdown. To estimate the relative stability of this species, chloramphenicol and rifampicin were added to the cultures after $75 \mathrm{~min}$ of transposase induction to block further transposase synthesis and the transcription, respectively, necessary for plasmid replication. The level of the figure-eight species (Fig. 9b, top) clearly falls rapidly with a half-life of $\sim 30 \mathrm{~min}$ (Fig. 9b, bottom). Similar results were obtained for induction times of 30 , 40 , and $60 \mathrm{~min}$ (data not shown). In all cases transposon circles accumulate to levels that correlate with the time of induction: these levels are significantly higher after 75 min induction than after $30 \mathrm{~min}$ (data not shown).

The immediate increase in levels of the figure-eight form with transposase induction and its immediate decrease after antibiotic addition, which is independant of the duration transposase induction, implies that its formation requires de novo synthesis of transposase. On the other hand, although circle formation also requires transposase, the effect of inhibition of transposase synthesis is delayed significantly. Although this does not constitute a formal proof, it is consistent with the hypothesis that figure-eight molecules are precursors of transposon circles.

\section{Discussion}

IS911 transposase catalyzes single-stranded DNA circularization: a one-ended site-specific transposition reaction

The results presented here demonstrate that the IS 911 transposase Orf $\mathrm{AB}$, when supplied in the absence of other IS911-encoded proteins, is capable of mediating specific circularization of a single transposon strand 

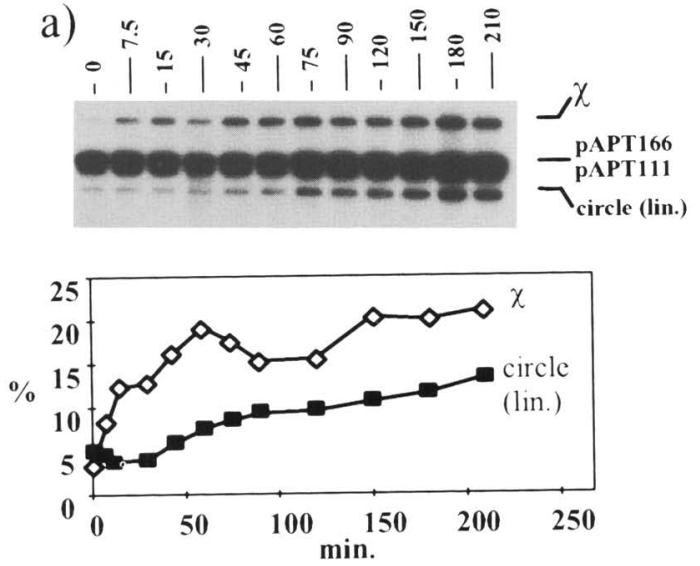

b)
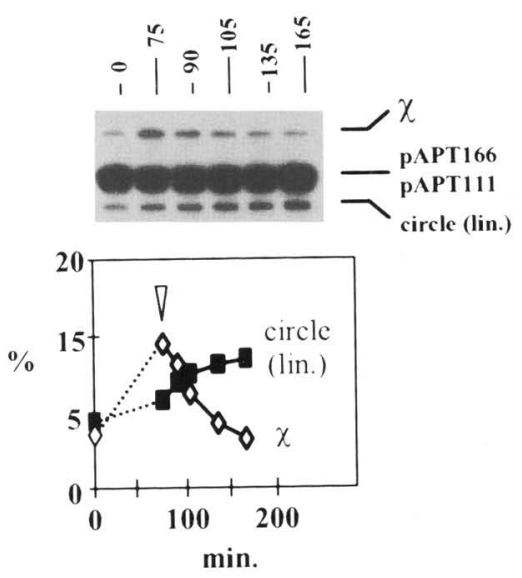

Figure 9. Kinetics of induction and chase of figure-eights and transposon circles. (a) Kinetics of induction. (Top) A Southern hybridization of plasmid DNA samples prepared at different times after induction of OrfAB synthesis, digested with XhoI, NdeI, and PstI, separated by electrophoresis on a $1 \%$ TAE-agarose gel and probed with oligonucleotide $\mathrm{O}_{2}$. This oligonucleotide hybridizes to transposon circles and figure-eight molecules in addition to DNA fragments derived from the transposase donor plasmid, pAPT111, and the wild-type transposon donor, pAPT166. The positions of the different DNA species are indicated at right. Numbers above indicate time in minutes after induction. (Bottom) A quantitative representation of the kinetics of appearance of the $\chi$ forms $(\diamond)$ and transposon circles (D) normalized to the sum of the pAPT111 and pAPT166 fragments in each lane. (b) Kinetics of disappearance of figure-eights and transposon circles. Symbols are identical to those in $a$. In the experiment shown, induction of transposase synthesis was for $75 \mathrm{~min}$ before addition of chloramphenicol and rifampicin (indicated by the open arrowhead at the bottom).

while leaving the second strand intact. Because the transposon donor molecule is a circular plasmid, the reaction generates an unusual figure-eight form.

The figure-eight species can be observed using a nondenaturing plasmid extraction method but is lost during the alkaline lysis procedure, presumably as a result of denaturation of the unjoined strand of the plasmid backbone. In this light it is perhaps surprising that the un- joined strand is apparently not degraded in vivo. This stability suggests that it might be inaccessible to exonuclease attack, perhaps as a result of continued transposase binding after the single-strand exchange. This type of protection has also been observed in vivo for transposon $\operatorname{Tn} 10$ (Haniford et al. 1991). It is important to note that although the figure-eight molecule carries a single-strand nick, its electrophoretic behavior and its appearance in the electron microscope indicates that it retains some supercoiling. As has proved to be the case for other site-specific recombination reactions (Stark et al. 1992), analysis and definition of the supercoiled state of the figure-eight should provide further information concerning the mechanism of synapsis and strand exchange.

The structure of the figure-eight is fully consistent with a mechanism in which transposase catalyzes a single-strand cleavage liberating a $3^{\prime} \mathrm{OH}$ transposon end and its transfer to a target $3 \mathrm{bp} 5^{\prime}$ to the opposite end. Therefore, the reaction can be viewed as one-ended site-specific transposition. This type of single-strand cleavage and transfer is analogous to the processing and integration reactions observed in vitro with purified retroviral integrases (IN; see Skalka 1993; Vink and Plasterk 1993). It is important to note that Orf $A B$ shares significant similarities with amino acids thought to lie within the active site of IN (see Polard and Chandler 1995). Furthermore, both the retroviral provirus and IS911 terminate with the same 5'-CA-3' dinucleotide. IN processes linear retroviral double-stranded DNA by a single-strand cleavage at each end that removes the two $3^{\prime}$ terminal bases. The 3' hydroxyl group liberated $\left(5^{\prime}-\mathrm{CA}-3^{\prime}-\mathrm{OH}\right)$ then serves as a nucleophile in attacking a suitable target DNA. This conserved dinucleotide is essential for endprocessing and strand transfer by IN in vitro and in vivo (see Vink and Plasterk 1993). In the results presented here it is demonstrated that mutation of the dinucleotide at both IS911 ends eliminates formation of figure-eight molecules, whereas mutation at one or the other end simply inhibits the transfer activity of the mutated end. It has yet to be determined whether, as in the retroviral case, the terminal dinucleotide is essential for the initial strand cleavage reaction.

Because the conserved dinucleotide is essential in vivo for the transfer of an IS911 end, this strongly supports the notion that the retroviral and IS911 strand transfer reactions occur using a similar mechanism catalyzed by "homologous" enzymes.

There are two important differences, however, between the known activities of IN in vitro and in vivo and those of the IS 911 transposase observed here in vivo. IN requires a free DNA end for its processing activity. Current evidence suggests that this reflects a requirement for "unpairing" of DNA bordering the site of cleavage (van den Ent et al. 1994; P. Brown, pers. comm.). In contrast, transposase necessarily catalyzes $3^{\prime}$ end cleavage at IS911 ends while they are embedded in genomic DNA. This may be attributable, at least in part, to the fact that OrfAB is specifically targeted to the ends of the transposon, whereas IN shows no obvious sequence-specific 
recognition of its substrate. It may also reflect a capacity of the transposase to locally "unpair" DNA either by itself or as a result of the elaboration of an ordered nucleoprotein transposition complex.

The second difference is that transposon strand transfer occurs in a site-specific fashion to generate the circularized transposon strand. This presumably results from specific synapsis of the two ends in an ordered nucleoprotein structure maintained by protein-protein interactions. Such structures have been described in the transposition of bacterial elements $\mathrm{Mu}, \operatorname{Tn} 10$, and $\operatorname{Tn} 7$ (Mizuuchi et al. 1992; Bainton et al. 1993; J. Sakai and N. Kleckner, pers. comm.). During transposition, the two specifically juxtaposed ends would be cleaved and transferred to a suitable target molecule. Figure-eight formation clearly reflects the absence of such an exogenous target. In this case, an uncleaved transposon end would be held in close enough proximity to the cleaved end to act as a target for $3^{\prime}$ strand transfer. Cleavage of the two ends cannot be concerted during figure-eight formation as $3^{\prime}$ strand cleavage of the opposite end without strand transfer would result in the formation of "lariat" structures. These have not been observed. Finally, because strand transfer of a mutated end cannot be detected but strand transfer to a mutated end is clearly observed, the results demonstrate that although the $5^{\prime}-\mathrm{CA}-3^{\prime}$ is essential for donor transfer, it is not essential for target function.

\section{Is the figure-eight form an intermediate state of circularization?}

It is tempting to speculate that the second product observed in the cells expressing the IS911 transposase alone, a transposon circle in which both transposon strands are circularized, is derived from figure-eight forms by a second strand exchange. If this is the case, circularization of the second strand cannot occur in an identical manner to that of the first strand. This is because mutation of the terminal dinucleotide at one end does not abolish production of excised transposon circles and, moreover, gives rise to a circle population in which the junction sequence is homogenous. Therefore, circularization of the second strand necessary to excise the element must involve a different mechanism, which remains to be established.

The kinetic data are consistent with the idea that the figure-eights are precursors of the circles; they are produced earlier than the circles and disappear immediately after inhibition of transposase synthesis. This suggests that figure-eights may be the product of a one-step reaction, and the delayed appearance of circles suggests that they may be produced in a second step. However, quantitative results from the chase experiments do not provide definitive proof that the figure-eights are converted into circles. Indeed, fewer circles appear to accumulate than would be predicted from the level of figure-eight molecules produced, although this may simply reflect an equilibrium between synthesis and breakdown of the circles that do show some capacity to insert into a suitable target molecule (Polard et al. 1994; B. Ton-Hoang, unpubl.). This might explain the slight decrease in levels of transposon circles measured at early times of transposase induction.

One clear implication of these results is that circle formation does not necessarily reflect a cut and paste mechansim in which the intermediate would be an excised linear form of the element generated by doublestrand cleavage at both ends. IS911 excision as a circle may, therefore, proceed by consecutive circularization of each strand.

\section{Relationship between figure-eight formation and transposition}

The results described above demonstrate that OrfAB alone has the capacity to catalyze a single-strand cleavage and transfer reaction. If reactions of this type occurred at both ends of the element and strand transfer took place into a suitable target, this would generate a branched intermediate proposed by Arthur and Sherratt (1979) and Shapiro (1979). The transposon would remain attached to the donor backbone by a single-strand joint at its $5^{\prime}$ ends and to the target by a single-strand joint at its $3^{\prime}$ ends. Resolution of this intermediate by replication of the transposable element would be expected to generate cointegrated molecules in which the donor and target replicons are fused and are separated at each junction by a copy of the transposable element in direct repeat. However, the major IS911 intermolecular transposition products are not cointegrate molecules but simple insertions (Polard et al. 1992, 1994). One way of explaining such a result is that an additional factor is necessary for $5^{\prime}$ cleavage, releasing the transposon from its donor molecule. A second is simply that the intermolecular transposition intermediate is the transposon circle. On such a substrate, one single-strand cleavage at each of the abutting ends would generate a linear molecule whose integration would give rise to a simple insertion. Circular molecules of this type are indeed capable of integrating into suitable targets (Polard et al. 1991, 1994; B. Hoang Tan, P. Polard and M. Chandler, unpubl.). It is important to note, however, that the level of transposon circles generated by OrfAB is modulated by concurrent production of OrfA in the host. The effect of OrfA is to reduce the appearance of circles and to stimulate intermolecular transposition (Polard et al. 1992). Therefore, OrfA "channels" Orf $A B$ recombination activity, possibly by direct intervention in the elaboration a synaptic complex involving the transposon ends and OrfAB. Circle and possibly figure-eight production may reflect recombination that has escaped the control of OrfA. Further detailed analysis of the recombination reactions catalyzed by OrfAB and the overall mechanism of IS 911 transposition must await the development of a defined in vitro system.

Finally, it should be noted that some other transposable elements are known to undergo circularization (Rose and Snutch 1984; Ruan and Emmons 1984; Jahn et al. 1989; Tausta and Klobutcher 1989; Williams et al. 1993; Sekine and Ohtsubo 1994). Interestingly, the pu- 
tative recombinases/transposases encoded by several of these elements and that would be involved in this excisive process, like the transposase of IS911, are characterized by the presence of a DD35E domain (Doak et al. 1994). It would be interesting to determine whether figure-eight molecules also accompany excision/circularization of these related elements.

\section{Materials and methods}

Bacterial strains, media, and compounds

The Escherichia coli host strain used in this study is IS219 [srlC::Tn10, recA1, malPp::lacl ${ }^{\mathrm{q}}$, araD139, S(ara leu), galU galK, hsdM, hsdS, rplsLD(lacIOPZYA|X74] (see Polard et al. 1992). Cells were grown at $37^{\circ} \mathrm{C}$ in Luria broth supplemented where necessary with ampicillin (Ap, $100 \mu \mathrm{g} / \mathrm{ml}$ ) and kanamy$\operatorname{cin}(\mathrm{Km}, 25 \mu \mathrm{g} / \mathrm{ml})$. IPTG $(100 \mu \mathrm{M})$ was added to induce the plac promoter used to drive OrfAB synthesis. Rifampicin (Rif, 250 $\mu \mathrm{g} / \mathrm{ml})$ and chloramphenicol $(\mathrm{Cm}, 140 \mu \mathrm{g} / \mathrm{ml})$ were added to block RNA and protein synthesis, respectively.

\section{Plasmids}

Plasmids carrying IS911-based transposons used in circularization assays

The artificial IS 911 -based transposon carrying the translational fusion orf $A-l a c Z$ was assembled as follows. The SmaI-BamHI fragment of pPLC1983 (Linder et al. 1985) carrying the coding sequence of $l a c Z$ was substituted for the DraI-BamHI fragment of pOFT139, carrying the major part of the IS911 sequence (Prère et al. 1989), to give pAPT35. This fuses the first 8 amino acids of OrfA to $\operatorname{lac} Z$ at its eighth codon. The plasmid retains IRL but is deleted for IRR. To introduce IRR downstream of the orf $A-1 a c Z$ fusion, the EcoRI-BamHI fragment of pAPT35, which includes IRL and the entire fusion, was substituted for the equivalent EcoRI-BglII fragment in pAPT72 (Polard et al. 1992) to give the pAPT166 (Fig.1).

Plasmids carrying IS 911 -based orf $A-1 a c Z$ fusion transposons with mutations of the terminal $5^{\prime}-\mathrm{CA}-3^{\prime}$ dinucleotide of IRL, IRR, or both, were constructed using pAPT99 (wild-type IRR and IRL), pAPT104 (mutated IRL), pAPT105 (mutated IRR), and pAPT108 (mutated IRL and IRR) (Polard et al. 1992). These carry IS911-based transposons in which copies of IRL and IRR flank a Cm-resistance gene. In pAPT104, pAPT105, pAPT108, IRR is bordered by a sequence from phage $\lambda$ that acts as a surrogate IS911 end (Polard et al. 1994). This sequence was eliminated either by substitution of IRR from PAPT99 into pAPT104 (to give pAPT177) or, in the case of pAPT105 and pAPT108, by direct deletion between the BamHI site located outside the transposon and a $B g l I I$ site marking the IRR mutation (to give pAPT178, and pAPT179, respectively). The Cm resistance gene of pAPT177, pAPT178 and pAPT179 was then replaced by the orf $A-$ lac $Z$ fusion of pAPT1 66 using the Ball site present in both IRL and IRR to give pAPT180 (mutated IRL), pAPT181 (mutated IRR), and pAPT182 (mutated IRL and IRR).

Plasmids used to drive IS911 protein expression in trans of IS911-based transposons

The BamHI-EcoRI fragment of pAPT1 (Polard et al. 1991) was replaced by a polylinker (BamHI-SmaI-KpnI-EcoRI) to give pAPT2. The EcoRI-PstI fragment of pAPT2 carrying the cI gene of phage $\lambda$ was then replaced by the EcoRI-Pst fragment of pMJ1560 (Stark et al. 1987) carrying the $1 a c I^{\mathrm{q}}$ allele to give
pAPT2.1 The plac promoter (with the L8 and UV5 mutations) was isolated from pBR793 (Calos 1978) as a PCR fragment with the two oligonucleotides 5'-CCAGATCTCGAGGAAAGCGGGCAGTGAGCG-3' and 5'-GCGGATCCTCTAGACCTGTGAAATTGTTATCCGC-3'. These introduce BgIII (bold) and $X$ hoI (italic), and $\mathrm{XbaI}$ (bold) and BamHI (italic) sites $5^{\prime}$ and $3^{\prime}$, respectively, to the promoter. The amplified fragment was digested with BglII and BamHI, cloned into the BamHI site of the polylinker of pAPT2.1, and its sequence was verified. In the resulting plasmid, pAPT2.2, transcription is directed from the $B a m H I$ site to the EcoRI site. In addition, four terminators of the $\operatorname{rrn} B$ operon of $E$. coli were introduced upstream of plac. These were included on a XhoI-BamHI fragment from pRS551 (Simons et al. 1987) carrying the 5' end of the Km marker also present on pAPT2.2. This fragment was substituted for a XhoIXhoI fragment of pAPT2.2. The BamHI extremity of the donor fragment and one of the $X h o I$ sites of the vector plasmid were made flush with T4 DNA polymerase and the fragment was cloned in an orientation that reconstitutes the $\mathrm{Km}$ gene to give pAPT2.3. The $\Omega$ fragment of pHP45 (Prentki and Krisch 1987) with a spectinomycin $(\mathrm{Sp})$ and a streptomycin $(\mathrm{Sm})$-resistance gene flanked by T4 gene 32 terminators was then cloned as a blunted BamHI fragment into a blunted EcoRI site located upstream of plac. Plasmid pAPT110 carries the fragment in an orientation in which the direction of transcription of the $\mathrm{SpSm}$ marker is identical to that directed by plac. Different combinations of IS 911 open reading frames orf $A$ and orf $B$ were finally placed under the control of plac. The native orf $A$ and orf $B$ sequence was cloned as a BamHI fragment of IS911 from pAPT10 (Polard et al. 1991) into the BamHI site of pAPT110. The fusedframe mutant was similarly cloned from pAPT93 (Polard et al. 1991). The BamHI site proximal to plac was destroyed by filling in with the T4 DNA polymerase to generate pAPT112 and pAPT111, respectively. In these expression plasmids the first 22 bp of IRL and IRR have been deleted to create an immobile IS911 derivative, also lacking part of the -35 sequence of the putative promoter located in IRL but retaining endogenous translational sequences (Prère et al. 1989).

\section{Transformation and cell growth}

Strains carrying different combinations of the transposon-donor and transposase-donor were constructed by successive transformations. The transposase-donor plasmids were introduced systematically after the transposon-donor plasmids.

Strains carrying both test plasmids were streaked on L agar supplemented with Ap or $\mathrm{Km}$, or both, when required, and incubated for $12 \mathrm{hr}$ at $37^{\circ} \mathrm{C}$. Fresh isolated colonies were then used to inoculate similarly supplemented $\mathrm{L}$ broth, with or without IPTG, and incubated overnight at $37^{\circ} \mathrm{C}$.

For kinetic analysis, overnight cultures grown without IPTG were used to inoculate fresh $\mathrm{L}$ broth supplemented with Ap and $\mathrm{Km}$ at an $\mathrm{OD}_{600}$ of 0.1 and the cultures were grown at $37^{\circ} \mathrm{C}$ to an $\mathrm{OD}_{600}$ of 0.5 , before IPTG addition. Growth was continued at $37^{\circ} \mathrm{C}$, and at various times after IPTG addition, a similar number of $\mathrm{OD}_{600}$ units were collected, mixed in two volumes of ice-cold TN [10 mM Tris (pH 7.5), $100 \mathrm{~mm} \mathrm{NaCl}]$, centrifuged (15 $\min$ at $5000 \mathrm{~g}, 4^{\circ} \mathrm{C}$ ), washed with $1 / 10$ volume of $\mathrm{TN}$, and kept on ice.

\section{Alkaline and cleared lysates}

Alkaline lysates were prepared as described by Birnboim and Doly (1979).

Cleared lysates were prepared essentially as described (Clewell and Helinski 1969), except that the cleared superna- 
tants were collected and incubated with proteinase $\mathrm{K}(0.5 \mathrm{mg} /$ $\mathrm{ml})$ in the presence of SDS $(0.5 \%)$ for $1 \mathrm{hr}$ at $50^{\circ} \mathrm{C}$. DNA was concentrated by isopropanol precipitation and resuspended in TE buffer.

\section{Synthetic oligonucleotides: sequence and phosphorylation}

The oligonucleotides $\left(5^{\prime} \rightarrow 3^{\prime}\right)$ used in the PCR amplification, sequence determination, and primer extension were $\mathrm{O}_{0}$ TATAAAAATAGGCGTATCACGAGGC; $\mathrm{O}_{2}$, GGTGAGCATATCACCTCTGTTCAGG; $\mathrm{O}_{3}$, ACTCTGCGCTGAAATTTCTTTTTTTC; $\mathrm{O}_{5}$, GATACTGGAAAAAACTCTAACTCGG; and $\mathrm{O}_{7}$, TCAACGCATATAGCGCTAGCAGCACG.

Radiolabeling was with 8 pmoles of oligonucleotides, 16 pmoles of $\left[\gamma^{32} \mathrm{P}\right]$ ATP $(5000 \mathrm{Ci} / \mathrm{mmole}$; Amersham), and 1 unit of T4 kinase (NEB, Inc.) in T4 kinase buffer (ob70 $\mathrm{mm}$ Tris- $\mathrm{HCl}$ (pH 7.6), $10 \mathrm{mM} \mathrm{MgCl}_{2}, 5 \mathrm{~mm}$ dithiothreitol] for $45 \mathrm{~min}$ at $37^{\circ} \mathrm{C}$.

\section{Gel electrophoresis and DNA quantification by hybridization}

Agarose gel electrophoresis was in TAE buffer at room temperature.

DNA hybridization was accomplished directly in the gel to avoid differential loss of DNA species because of differences in transfer efficiency. The gel was dried for $1 \mathrm{hr}$ at $50^{\circ} \mathrm{C}$. The DNA was denatured $(0.5 \mathrm{M} \mathrm{NaOH}, 0.15 \mathrm{M} \mathrm{NaCl}, 20 \mathrm{~min})$, and the gel neutralized in $0.5 \mathrm{M}$ Tris $(\mathrm{pH} 8), 0.15 \mathrm{M} \mathrm{NaCl}$. After a prehybridization step in $6 \times \mathrm{SSC}, 0.5 \%$ SDS, $100 \mathrm{mg} / \mathrm{ml}$ of calf thymus DNA and $0.1 \%$ nonfat milk for $6-8 \mathrm{hr}$, hybridization was carried out with a 20-fold excess of labeled olignucleotide for 12-15 $\mathrm{hr}$ in the same buffer. Both steps were performed at $8^{\circ} \mathrm{C}$ under the melting temperature of the probe oligonucleotide. The gel was then washed three times in $6 \times$ SSC, $0.1 \%$ SDS, at room temperature, dried quickly, and exposed either for autoradiography using Kodak XR5 film or, for quantification, in a Fuji X BAS1000 PhosphorImaging system coupled to the PCBas software.

\section{Polymerase chain reaction}

The PCR was performed on $10 \mathrm{ng}$ of purified template in a final volume of $50 \mu \mathrm{l}$ with $100 \mu \mathrm{M}$ of each dNTP, l unit of Tub polymerase, and 10 pmoles of each primer as recommended (Amersham). Twenty-five cycles of $1 \mathrm{~min}\left(94^{\circ} \mathrm{C}\right), 2 \min \left(16^{\circ} \mathrm{C}\right.$ under the lowest melting temperature of the two primers), and $2 \min \left(72^{\circ} \mathrm{C}\right)$, were used. DNA was ethanol precipitated before analysis by agarose gel electrophoresis.

\section{Gel purification of DNA, sequencing, and primer extension}

Purification of the figure-eight molecule, the $\alpha$ and of the $x$ forms from the agarose gels, was as for the circle (Polard et al. 1992). DNA sequences were determined from $3 \mu \mathrm{g}$ of purified figure-eight molecules with a T7 sequencing kit (Pharmacia) as recommended.

Primer extension used $150 \mathrm{ng}$ of purified figure-eight (digested by BalI), 0.1 pmole of ${ }^{32} \mathrm{P}$ phosphorylated $\mathrm{O}_{0}, 0.5$ unit of Tub DNA polymerase, and a final concentration of $500 \mu \mathrm{M}$ of each $\mathrm{dNTP}$ in a final volume of $15 \mu \mathrm{l}$. The single cycle of $1 \mathrm{~min}$ $\left(94^{\circ} \mathrm{C}\right), 2 \mathrm{~min}\left(16^{\circ} \mathrm{C}\right.$ under the melting temperature of the oligonucleotide), and $3 \mathrm{~min}$ at $72^{\circ} \mathrm{C}$ was stopped by the addition of $5 \mu \mathrm{l}$ of $80 \%$ formamide, $10 \mathrm{~mm}$ EDTA, $10 \mathrm{~mm} \mathrm{NaOH}, 0.1 \%$ bromophenol blue. One-quarter of the volume was resolved on an $8 \% \mathrm{TBE} /$ acrylamide/urea gel. Sequence reactions to serve as markers were by thermal cycling (Amersham) on pAPT166 using the same oligonucleotide.

Direct extension was performed with Tub DNA polymerase (0.5 unit) on $150 \mathrm{ng}$ of purified figure-eight molecules derived from single-end mutants and $300 \mathrm{ng}$ for the wild-type transposon. The substrates (digested with $B a l I$ and $E c o$ RI) were incubated with dATP, dCTP, and dGTP each at $500 \mu \mathrm{M}$, dTTP (100 $\mu \mathrm{M}), 5 \mu \mathrm{Ci}$ of $\left[\alpha^{-}{ }^{32} \mathrm{P}\right] \mathrm{dTTP}(800 \mathrm{Ci} / \mathrm{mmole}$; Amersham $)$ in a final volume of $15 \mu \mathrm{l}$. The single cycle of extension and the analysis of the sample were as described above. A sequence ladder of M13mp18 was generated using a T7 sequencing kit (Pharmacia) as indicated by the supplier.

\section{Acknowledgments}

We thank members of the Mobile Genetic Elements group for discussions and advice during the course of this work. Special thanks go to E. Boy de la Tour and L. Caro for the electron microscopy, and to $M$. Bétermier for stimulating comments throughout. We also acknowledge E. Käs for critically reading the manuscript, and P. Brown, N. Kleckner, and J. Sakai for communicating their results before publication. We apologize to our colleagues in the retroviral integration field for not citing individual contributions. This is because of the extensive literature and space limitations. These references can be found in the review articles cited. This work has benefited from grants from the CNRS (UPR9007), the Association pour la Recherche contre le Cancer (6406), the Ligue Nationale contre le Cancer (bequest Petijean), and an European Union network grant in the Human Capital and Mobility program.

The publication costs of this article were defrayed in part by payment of page charges. This article must therefore be hereby marked "advertisement" in accordance with 18 USC section 1734 solely to indicate this fact.

\section{References}

Arthur, A. and D.J. Sherratt. 1979. Dissection of the transposition process: A transposon-encoded site-specific recombination system. Mol. \& Gen. Genet. 175: 267-274.

Bainton, R.J., K.M. Kubo, J.N. Feng, and N.L. Craig. 1993. Tn7 transposition: Target recognition is mediated by multiple Tn7-encoded proteins in a purified in vitro system. Cell 72: 931-943.

Birnboim, H.C. and J. Doly. 1979. A rapid alkaline extraction DNA procedure for screening recombinant plasmid DNA. Nucleic Acids Res. 7: 1513-1523.

Calos, M.P. 1978. DNA sequence for a low level promoter of the lac repressor gene and "up" promoter mutation. Nature 274: 762-765.

Caparon, M.G. and J.R. Scott. 1989. Excision and insertion of the conjugative transposon Tn916 involves a novel recombination mechanism. Cell 59: 1027-1034.

Clewell, D.B. and D.R. Helinski. 1969. Supercoiled circular DNA-protein complex in Escherichia coli: Purification and induced conversion to an open circular DNA form. Proc. Natl. Acad. Sci. 62: 1159-1166.

Doak, T.G., F.P. Doerder, C.L. Jahn, and G. Herrick. 1994. A proposed superfamily of transposase genes: Transposon-like elements in ciliated protozoa and a common "D35E" motif. Proc. Nat1. Acad. Sci. 91: 942-946.

Fayet, O., P. Ramond, P. Polard, M.F. Prère, and M. Chandler. 1990. Functional similarities between retroviruses and IS3 family of bacterial insertion sequences? Mol. Microbiol. 4: 1771-1777. 
Haniford, D.B., H.W. Benjamin, and N. Kleckner. 1991. Kinetic and structural analysis of a cleaved donor intermediate and a strand transfer intermediate in Tn10 transposition. Cell 64: 171-179.

Jahn, C.L., M.F. Krikauand, and S. Shyman. 1989. Developmentally coordinated en masse excision of a highly repetitive element in E. crassus. Cell 59: 1009-1018.

Jaraczewski, J.W. and C.L. Jahn. 1993. Elimination of Tec elements involves a novel excision process. Genes \& Dev. 7: 95-105.

Klobutcher, L.A., L.R. Turner, and J. LaPlante.1993. Circular forms of developmentally excised DNA in Euplotes crassus have a heteroduplex junction. Genes \& Dev. 7: 84-94.

Linder, P., G. Churchward, X. Guixian, Y. Yi-Yi, and L. Caro. 1985. An essential replication gene, repA, of plasmid pSC101 is autoregulated. J. Mol. Biol. 181: 383-393.

Mizuuchi, K. 1992. Transpositional recombination: Mechanistic insights from studies of $\mathrm{Mu}$ and other elements. Annu. Rev. Biochem. 61: 1011-1051.

Mizuuchi, M., T.A. Baker, and K. Mizuuchi. 1992. Assembly of the active form of the transposase-Mu DNA complex: A critical point in $\mathrm{Mu}$ transposition. Cell 70: 303-311.

Morisato, D. and N. Kleckner. 1984. Transposase promotes double strand breaks and single strand joints of Tn 10 termini in vivo. Cell 39: 181-190.

Polard, P. and M. Chandler. 1995. Bacterial transposases and retroviral integrases. Mol. Microbiol. 15: 13-23.

Polard, P., M.F. Prère, M. Chandler, and O. Fayet. 1991. Programmed translational frameshifting and initiation at an AUU codon in gene expression of bacterial insertion sequence IS911. I. Mol. Biol. 222: 465-477.

Polard, P., M.F. Prère, O. Fayet, and M. Chandler. 1992. Transposase-induced excision and circularization of the bacterial insertion sequence IS911. EMBO I. 11: 5079-5090.

Polard, P., L. Seroude, M.F. Prère, O. Fayet, and M. Chandler. 1994. One-ended insertion of IS911. J. Bacteriol. 176: 11921196.

Prentki, P. and H.M. Krisch. 1987. in vitro insertional mutagenesis with a selectable DNA fragment. Gene 29: 303-313.

Prère, M.F., M. Chandler, and O. Fayet. 1989. Transposition in Shigella dysenteriae: Isolation and analysis of IS911, a new member of the IS3 group of insertion sequences. I. Bacteriol. 172: 4090-4099.

Radice, A.D. and S.W. Emmons. 1993. Extrachromosomal circular copies of the transposon Tcl. Nucleic Acids Res. 21: 2663-2667.

Rose, A.M. and T.P. Snutch. 1984. Isolation of the closed circular form of the transposable element Tcl in Caenorhabditis elegans. Nature 311: 485-486.

Ruan, K. and S.W. Emmons. 1984. Extrachromosomal copies of transposon $\mathrm{Tcl}$ in the nematode Caenorhabditis elegans. Proc. Natl. Acad. Sci. 81: 4018-4022.

Schwartz, E., M. Kroger, and B. Rack. 1988. IS150: Distribution, nucleotide sequence, and phylogenetic relationships of a new E. coli insertion element. Nucleic Acids Res. 16: 67896802.

Scott, J.R., P.A. Kirchman, and M.G. Caparon. 1988. An intermediate in transposition of the conjugative transposon Tn916. Proc. Nat1. Acad. Sci. 85: 4809-4813.

Sekine, Y. and E. Ohtsubo. 1994. Translational control in production of transposase and in transposition of insertion sequence IS3. I. Mol. Biol. 235: 1406-1420.

Shapiro, J.A. 1979. Molecular model for the transposition and replication of bacteriophage $\mathrm{Mu}$ and other transposable elements. Proc. Natl. Acad. Sci. 76: 1933-1937.

Simons, R.W., F. Houman, and N. Kleckner. 1987. Improved single and multicopy lac-based cloning vectors for protein and operon fusions. Gene 53: 85-87.

Skalka, A.M. 1993. Retroviral DNA integration: Lessons for transposon shuffling. Gene 135: 175-182.

Stark, M.J.R. 1987. Multicopy expression vectors carrying the lac repressor gene for regulated high-level expression of genes in Escherichia coli. Gene 51: 255-267.

Stark, W.M., Boocock, M.R., and D.J. Sherratt. 1992. Catalysis by site-specific recombinases. Trends Genet. 8: 432-437.

Sundaresan, V. and M. Freeling. 1987. An extrachromosomal form of the Mu tramposons of maize. Proc. Natl. Acad. Sci. 84: 4924-4928.

Tausta, S.L. and L.A. Klobutcher. 1989. Detection of circular forms of eliminated DNA during macronuclear development in E. crassus. Cell 59: 1019-1026.

van den Ent, F.M., C. Vink, and R.H.A. Plasterk. 1994. DNA substrate requirements for different activities of the human immunodeficiency virus type 1 integrase protein. I. Virol. 68: 7825-7832.

van Luenen, H.G.A.M., S.D. Colloms, and R.H.A. Plasterk. 1993. Mobilization of quiet, endogenous Tc3 transposons of Caenorhabditis elegans by forced expression of $\mathrm{Tc} 3$ transposase. EMBO I. 12: 2513-2520.

Vink, C. and R.H.A. Plasterk. 1993. The human immunodeficiency virus integrase protein. Trends Genet. 9: 433-437.

Vögele, K., E. Schwartz, C. Welz, and B. Rak. 1991. High-level ribosomal frameshifting directs the synthesis of IS150 gene products. Nucleic Acids Res. 19: 4377-4385.

Williams, K., T.G. Doak, and G. Herrick. 1993. Developmental precise excision of Oxytrichia trifallax telomere-bearing elements and formation of circles closed by a copy of the flanking target duplication. EMBO J. 12: 4593-4601. 


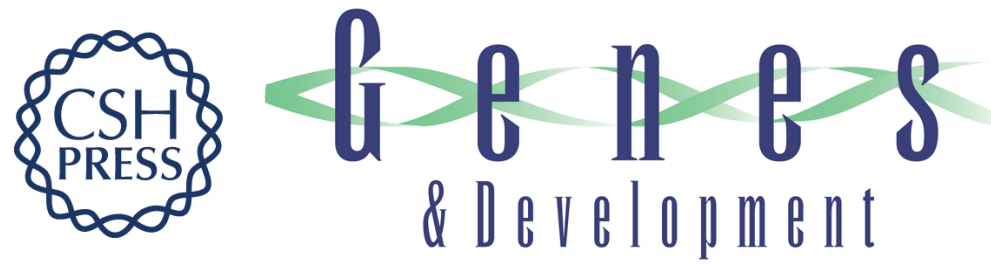

\section{An in vivo transposase-catalyzed single-stranded DNA circularization reaction.}

P Polard and M Chandler

\section{Genes Dev. 1995, 9:}

Access the most recent version at doi:10.1101/gad.9.22.2846

References This article cites 40 articles, 10 of which can be accessed free at:

http://genesdev.cshlp.org/content/9/22/2846.full.html\#ref-list-1

\section{License}

Email Alerting

Service

Receive free email alerts when new articles cite this article - sign up in the box at the top right corner of the article or click here.

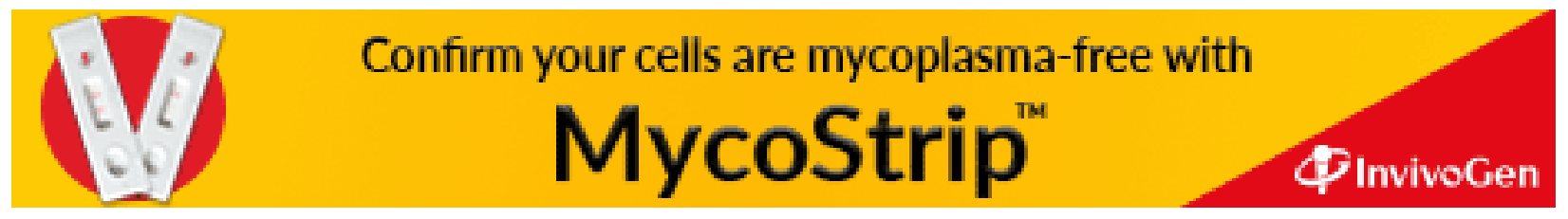

